\title{
A propósito de un caso de neumonía por Arcanobacterium pyogenes
}

\author{
A. HERMIDA AMEIJEIRAS, P. ROMERO JUNG ${ }^{1}$, A. CABARCOS ORTIZ DE \\ BARRÓN, M. TREVIÑO CASTALLO ${ }^{1}$
}

Servicios de Medicina Interna y Microbiología y ${ }^{1}$ Parasitología. Complejo Hospitalario de Santiago. Santiago de Compostela

ONE CASE OF PNEUMONIA WITH ARCANOBACTERIUM PYOGENES

\begin{abstract}
RESUMEN
Arcanobacterium pyogenes es un bacilo anaerobio Gram positivo perteneciente a la flora habitual de muchos animales domésticos. Las infecciones por Arcanobacterium pyogenes son extremadamente infrecuentes en humanos y la mayoría de casos publicados son cuestionados dado que no consiguen identificar claramente al patógeno.

Presentamos el caso de un varón sin antecedentes clínicos de interés con infección respiratoria y hemocultivos positivos confirmados para Arcanobacterium pyogenes. Fue necesaria la combinación de claritromicina y cefotaxima para conseguir la curación del paciente.

Este caso muestra la capacidad del A. pyogenes para provocar infecciones severas en humanos incluso en ausencia de enfermedades predisponentes.
\end{abstract}

PALABRAS CLAVE: Arcanobacterium pyogenes. Neumonía. Zoonosis

\begin{abstract}
Arcanobacterium pyogenes is an anaerobic Gram-positive bacilli and it is a part of the normal flora in many domestic animals. A. pyogenes is a rare cause of pyogenic infections in humans and most of cases reported are questionable since there was failure to definitively identify the pathogen.

A man with no past medical history presented with respiratory infection. The blood sample collected confirmed an Arcanobacterium pyogenes. It was necessary the addition of clarytromycin to the initial empirical treatment with cefotaxime, to the complete recovery.

This case provide further evidence that A. pyogenes can be a pathogen in humans even in absence of predisposing illness.
\end{abstract}

KEY WORDS: Arcanobacterium pyogenes. Pneumonia. Zoonosis.

Hermida Ameijeiras A, Romero Jung P, Cabarcos Ortiz de Barrón A, Treviño Castallo M. A propósito de un caso de neumonía por Arcanobacterium pyogenes. An Med Interna (Madrid) 2004; 21: 334-336.

\section{INTRODUCCIÓN}

Arcanobacterium pyogenes es un bacilo anaerobio gram positivo, no formador de esporas (1), que inicialmente estuvo emplazado en el género Actinomyces hasta el año 1997 en que fue incluido en el género Arcanobacterium (2), que podría traducirse como bacteria enigmática. Es colonizador habitual de la flora de muchos animales domésticos y origen de infecciones en estas mismas especies como neumonías, artritis sépticas o mastitis (3-5). Las infecciones en humanos por A. pyogenes son infrecuentes y la mayor parte de casos publicados no han aportado suficientes evidencias que permitan diferenciar claramente entre el A. pyogenes y el Arcanobacterium haemolyticum, este último implicado en un amplio espectro de enfermedades en humanos siendo la más frecuente, la faringoamigdalitis (6). Tan sólo cuatro de las series publicadas acerca de infecciones en humanos causadas por A. pyoge- nes, han sido confirmadas microbiológicamente. Éstas incluyen bacteriemias, una epidemia de sobre infección ulcerosa en niños tailandeses, otitis, cistitis, infecciones abdominales y abscesos (revisado por Drancourt y cols.) (7). No hemos encontrado bibliografía en la que se muestren casos de infecciones de vías respiratorias bajas por A. pyogenes así como ningún caso publicado de infección en humanos por este germen en nuestro país.

Presentamos el caso de un varón previamente sano con neumonía por Arcanobacterium pyogenes.

\section{CASO APORTADO}

Varón de 81 años, sin antecedentes médicos de interés, que ingresó en el mes de enero de 2003 por infección respiratoria. Vivía en el medio rural desde hacía 26 años donde trabajaba como granje-

\section{Trabajo aceptado: 4 de marzo de 2004}


ro. En los últimos ocho días previos al ingreso presentó disnea y tos productiva. En la exploración física destacaba $\mathrm{T}^{\mathrm{a}}$ ax. de $38,5^{\circ} \mathrm{C}$, ligeramente taquipneico y disminución del nivel de conciencia. Exploraciones complementarias: analítica: leucocitosis (12,2 x 10\%/l; con $86 \%$ neutrófilos y $3 \%$ cayados). Gasometría arterial: hipoxemia e hipocapnia $\left(\mathrm{pO}_{2}: 59 \mathrm{mmHg} ; \mathrm{pCO}_{2}: 25,7 \mathrm{mmHg}\right)$. $\mathrm{Rx}$ tórax: presencia de calcificaciones pleurales sin otros hallazgos de interés.

Inicialmente el tratamiento antibiótico empírico consistió en cefotaxima: $6 \mathrm{~g} / \mathrm{día}$. Tras seis días de tratamiento, el paciente no mostró mejoría y las muestras para hemocultivos retiradas el día del ingreso evidenciaron el crecimiento de Arcanobacterium pyogenes resistente a trimetroprim-sulfametoxazol. El TAC torácico mostró la presencia de derrame pleural bilateral e infiltrados pulmonares basales además de las calcificaciones pleurales inicialmente halladas en la Rx (Fig. 1). El cultivo de exudado faríngeo fue positivo para Candida tropicalis y Staphilococcus coagulasa negativo. No se hallaron otros datos de interés en el resto de exploraciones complementarias como ecografía abdominal, TAC cerebral y broncoscopia. El tratamiento antibiótico empírico inicial se cambió por cefotaxima 6 grs./día y claritromicina $1 \mathrm{~g}$ /día, obteniéndose la recuperación completa del paciente tras 8 días del nuevo tratamiento.

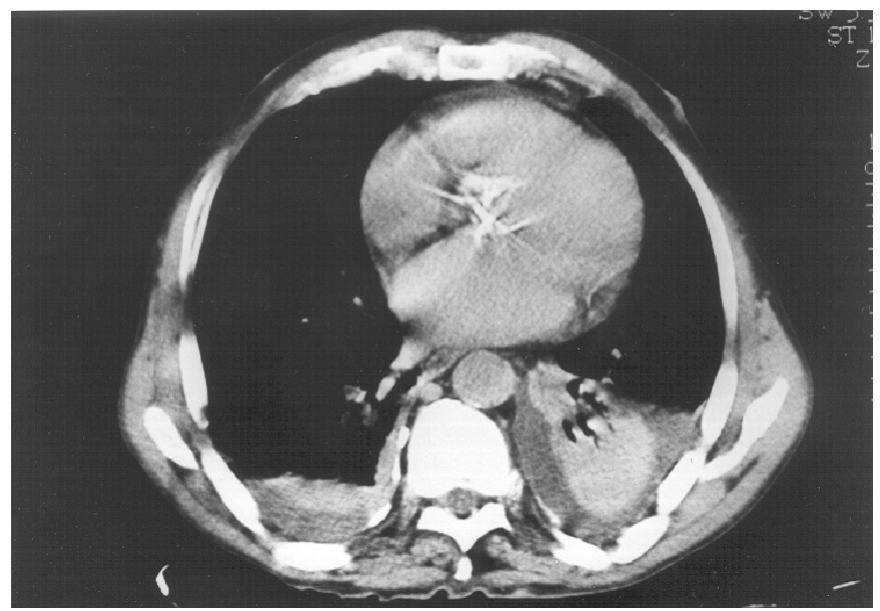

Fig. 1. TAC torácico mostrando derrame pleural bilateral con infiltrados pulmonares bibasales.

\section{HALLAZGOS MICROBIOLÓGICOS}

Al tercer día de incubación de las muestras sanguíneas, todos los hemocultivos anaeróbicos (BacT/Alert blood culture system; Organon Teknika Corporation, Durham, N.C.) evidenciaron el crecimiento de un bacilo gram positivo pleomórfico. La bacteria se aisló en agar sangre 5\% (Schaedler, bioMèrieux, France), tratándose de un organismo anaerobio facultativo cuyo crecimiento se veía potenciado si se cultivaba en una atmósfera de $\mathrm{CO}_{2}$ al 5-10\%, mostrando además poca capacidad de beta hemólisis. Se trataba de un bacilo gram positivo, no acidófilo, inmóvil y catalasa negativo. En el Api Coryne test (API System, bioMèrieux, France) provocaba la fermentación de la xilosa, maltosa y lactosa., hidrolizaba la gelatina así como poseía beta-glucuronidasas, alfa-glucosidasas y beta-galactosidasas. No hidrolizaba sin embargo, la esculina, y las reacciones de fermentación fueron negativas para la manosa y la sacarosa. El CAMP test mostraba una ligera potenciación de la hemólisis inducida por Staph. aureus. Otros test complementarios llevados a cabo y que resultaron positivos fueron el de la pirazinamidasa (API System, bioMèrieux, France) y la aglutinación con anticuerpos frente al Streptococo del grupo G (Slidex Streptokit, bioMèrieux, France). El test de resistencia antimicrobiana se llevó a cabo mediante el E-test (AB Biodisk, Solna, Sweden) en medio Müeller Hinton en atmósfera de $\mathrm{CO}_{2}$ al
5-10\% durante 48 horas. Los resultados del antibiograma se muestran en la tabla I.

Otras muestras obtenidas del paciente como raspado cutáneo o exudado faríngeo o aspirado bronquial, fueron cultivadas, resultando todas ellas negativas para $A$. pyogenes.

\section{TABLA I}

\section{SUSCEPTIBILIDADES ANTIMICROBIANAS IN VITRO DE LOS} HEMOCULTIVOS AISLADOS

\begin{tabular}{lcc}
\hline Antibiótico & CMI & S/I/R \\
Penicilina G & 0,125 & $\mathrm{~S}$ \\
Meticilina & 0,5 & $\mathrm{~S}$ \\
Amoxicilina/clavulánico & 0,047 & $\mathrm{~S}$ \\
Cefazolina & & $\mathrm{S}$ \\
Cefoxitina & 0,125 & $\mathrm{~S}$ \\
Cefotaxima & 0,38 & $\mathrm{~S}$ \\
Imipenem & 0,094 & $\mathrm{~S}$ \\
Gentamicina & 3 & $\mathrm{~S}$ \\
Eritromicina & & $\mathrm{S}$ \\
Clindamicina & & $\mathrm{S}$ \\
Azitromicina & 0,094 & $\mathrm{~S}$ \\
Claritromicina & 0,023 & $\mathrm{~S}$ \\
Trimethoprim-sulfametoxazol & & $\mathrm{R}$ \\
Tetraciclina & & $\mathrm{S}$ \\
Vancomicina & 0,38 & $\mathrm{~S}$ \\
Rifampina & & $\mathrm{S}$ \\
Cotrimoxazol & & $\mathrm{S}$ \\
Ciprofloxacino & 1 & $\mathrm{~S}$ \\
Linezolid & 0,19 & $\mathrm{~S}$
\end{tabular}

CMI: concentración mínima inhibitoria. S/I/R: sensible/intermedia/resistente.

\section{DISCUSIÓN}

Arcanobacterium pyogenes es un patógeno oportunista en animales domésticos, donde forma parte de la flora habitual de los tractos respiratorio alto, urogenital y gastrointestinal $(8,9)$. Los casos publicados de infecciones en humanos son muy escasos y la mayoría de ellos sin evidencia suficiente para poder diferenciarlo del Arcanobacterium haemolyticum (10). No forma parte de la flora habitual en el ser humano y la vía de transmisión desde los animales se desconoce, aunque en una epidemia de sobre infección de úlceras cutáneas por A. pyogenes, en niños escolares de Thailandia, se ha involucrado un tipo de moscas orientales como posible vector (11).

Parece que hay pocos métodos suficientemente fiables para distinguir entre el $A$. pyogenes y el A. haemolyticum. De hecho, ambas especies se clasificaron dentro del género Actinomices durante muchos años y sólo recientemente han sido asignados a un nuevo género (2). La identificación definitiva y diferenciación con respecto al $A$. haemolyticus se basa en la capacidad del primero para hidrolizar la gelatina en 48 horas de incubación, la fermentación de la D-xilosa, la producción de beta-glucuronidasas y la reacción positiva frente a anticuerpos contra Streptococo del grupo $\mathrm{G}$ así como una débil reacción frente a anticuerpos contra Streptococo del grupo B (12). Por otra parte, el A. pyogenes no incrementa la hemólisis llevada a cabo por los Streptococos del grupo B y potencia ligeramente la hemólisis llevada a cabo por el Staphilococo aureus (12). 
En este caso y al igual que sucedía en otros casos aportados anteriormente, el paciente estaba expuesto con frecuencia a patógenos procedentes de animales domésticos y aunque desconocemos cómo pudo contraer la infección por A. pyogenes, parece probable que se trate de una zoonosis. También merece la pena recalcar la aparente susceptibilidad in vitro del germen frente a beta-lactámicos que no se corresponde con los resultados in vivo, donde en

\section{Bibliografía}

1. Jousimies H and Summanen P. Microbiology Terminology Update: Clinically significant Anaerobic Gram-Positive and Gram-Negative Bacteria (Excluding Spirochetes). Clin Infect Dis 1997; 25: 11-4.

2. Smego R A, Jr. and Foglia G. Actinomycosis. Clin Infect Dis 1998; 26: 1255-63.

3. Smith JE. Corynebacterium species as animal pathogens. J Appl Bacteriol 1967; 29: 119-130.

4. Sorensen GH. Corynebacterium pyogenes: a biochemical and serological study. Acta Vet Scand 1974; 15: 544-554.

5. Gulbahar M and Gurturk K. Pyothorax associated with a Mycoplasma sp and Arcanobacterium pyogenes in a kitten. Aust Vet J 2002; 80: 344345.

6. Miller RA, Brancato F and Holmes KK. Corynebacterium hemolyticum as a cause of pharyngitis and scarlatiniform rash in young adults. Ann Intern Med 1986; 105:867-72.

7. Drancourt M, Oulès $\mathrm{O}$, Bouche $\mathrm{V}$ and Peloux $\mathrm{Y}$. Two cases of Actinomyces pyogenes Infection in Humans. Eur J Clin Microbiol Infect Dis 1993; 12: 55-57.

8. Narayanan S, Nagaraja TG, Wallace N, Staats M, Chengappa M and este caso ha sido necesario la utilización de macrólidos para lograr la erradicación de la infección. Este fenómeno también ha sido observado en infecciones por A. haemolyticus (13).

Aportamos el caso de un hombre previamente sano con neumonía y hemocultivos positivos para A. pyogenes, evidenciando el potencial patógeno del germen en humanos así como la severidad de las patologías implicadas.
Oberst RD. Biochemical and ribotypic comparison of Actinomyces pyogenes and A. pyogenes-like organisms from liver abscesses, ruminal wall and ruminal contents of cattle. Am J Vet Res 1998; 59: 271-276.

9. Timoney, JF, Gillespie JH, Scott FW and Barlough JE. Hagan and Brunner's microbiology and infectious diseases of domestic animals, 8th ed. Cornell University Press., Ithaca, N.Y.

10. Gahrn-Hansen B and Frederiksen W. Human Infections with Actinomyces pyogenes (Corynebacterium pyogenes). Diagn Microbiol Infect Dis 1992; 349-354.

11. Kotrajaras R, and Tagami H. Corynebacterium pyogenes- its pathogenic mechanism in epidemic leg ulcers in Thailand. Int J Dermatol 1987; 26: 45-50.

12. Lammler $\mathrm{C}$ and Blobet $\mathrm{H}$. Comparative studies on Actinomices pyogenes and Arcanobacterium haemolyticum. Med Microbiol Inmunol 1998; 177: 109-114.

13. Karpathios T, Drakonaki S, Zervoudaki A, Coupari G, Fretzatas A, Kremastinos $\mathrm{J}$ and Thomaidis. Arcanobacterium haemolitycum in oral presumed stretococcal pharyngotonsilitis or scarlet fever. J Pediatr 1992; 121: $735-7$. 